\title{
LA-UR-. $01-5063$
}

Approved for public release;

distribution is unlimited.

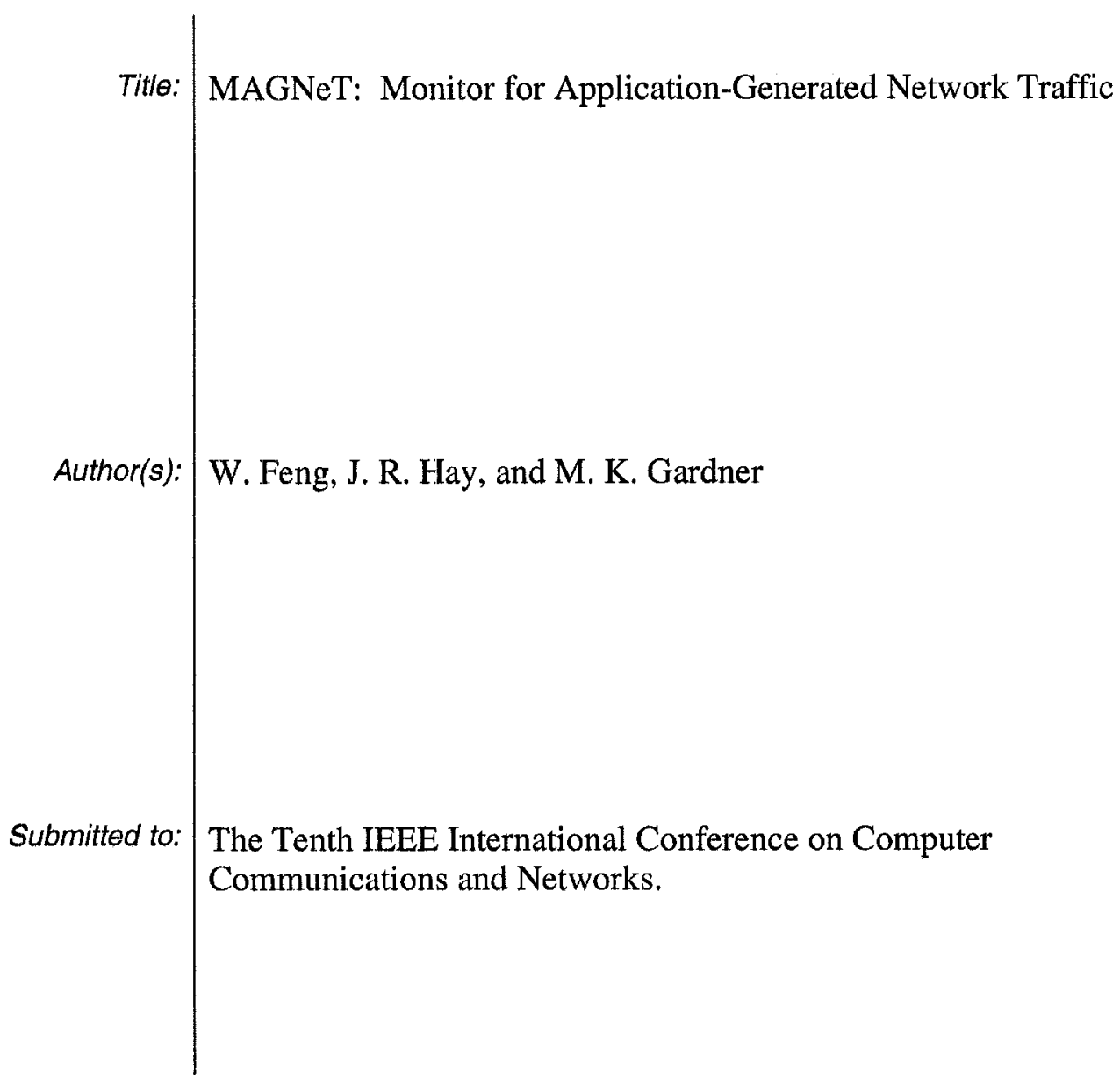

\section{Los Alamos}

NATIONAL LABORATORY

Los Alamos National Laboratory, an affirmative action/equal opportunity employer, is $\bar{\equiv}$ ted by the University of California for the U.S. Department of Energy under contract W-7405-ENG-36. By acceptance of this article, ublisher recognizes that the U.S. Government retains a nonexclusive, royalty-free license to publish or reproduce the published form of this contribution, or to allow others to do so, for U.S. Government purposes. Los Alamos National Laboratory requests that the publisher identify this article as work performed under the auspices of the U.S. Department of Energy. Los Alamos National Laboratory strongly supports academic freedom and a researcher's right to publish; as an institution, however, the Laboratory does not endorse the viewpoint of a publication or guarantee its technical correctness. 


\title{
MAGNeT: Monitor for Application-Generated Network Traffic
}

\author{
Wu-chun Feng, Jeffrey R. Hay, Mark K. Gardner \\ \{feng, jrhay, mkg\}@ianl.gov \\ Computer \& Computational Sciences Division \\ Los Alamos National Laboratory \\ Los Alamos, NM 87545
}

\begin{abstract}
Over the last decade, network practitioners have focused on monitoring, measuring, and characterizing traffic in the network to gain insight into building critical network components (from the protocol stack to routers and switches to network interface cards). Recent research shows that additional insight can be obtained by monitoring traffic at the application level (i.e., before application-sent traffic is modulated by the protocol stack) rather than in the network (i.e., after it is modulated by the protocol stack).

Consequently, this paper describes a Monitor for ApplicationGenerated Network Traffic (MAGNeT) that captures traffic generated by the application rather than traffic in the network. MAGNeT consists of application programs as well as modifications to the standard Linux kernel. Together, these tools provide the capability of monitoring an application's network behavior and protocol state information in production systems. The use of MAGNe'T will enable the research community to construct a library of real traces of application-generated traffic from which researchers can more realistically test network protocol designs and theory. MAGNeT can also be used to verify the correct operation of protocol enhancements and to troubleshoot and tune protocol implementations.
\end{abstract}

\section{MOTIVATION}

Although monitoring [1-6] and characterizing [7-10] traffic in the network provides insight into building critical network components (e.g., ideal buffer sizes in routers), recent research [11-13] shows that additional insight can be obtained by monitoring and measuring traffic at the application level (i.e., before it is modulated by the protocol stack) rather than in the network (i.e., after it is modulated by the protocol stack). For example, knowing application traffic patterns can provide insight into the design of a better protocol stack.

\section{A. Background}

Network researchers often use traffic libraries such as tcplib [14], network traces such as those at $[15,16]$, or network models such as those found in [9] to drive their network experiments, particularly to test the performance of network-protocol enhancements. However, such libraries, traces, and models are based on measurements made by tcpdump [1] (or similar tools like PingER [2], NLANR Network Analysis Infrastructure [4], NIMI [5], CoralReef [6]), meaning that the traffic an application sends on the network is captured only after having passed through TCP (or more generally, any protocol stack) and into the network. That is, the tools capture traffic on the wire (or in the network) rather than at the application level. Thus, the above tools cannot provide any protocol-independent insight into the actual traffic patterns of an application.

This work was supported by the U.S. Dept. of Energy's Laboratory-Directed Research \& Development Program and the Los Alamos Computer Science Institute through Los Alamos National Laboratory contract W-7405-ENG-36. Any opinions, findings, and conclusions, or recommendations expressed in this material are those of the author(s) and do not necessarily reflect the views of DOE, Los Alamos National Laboratory, or the Los Alamos Computer Science Institute.

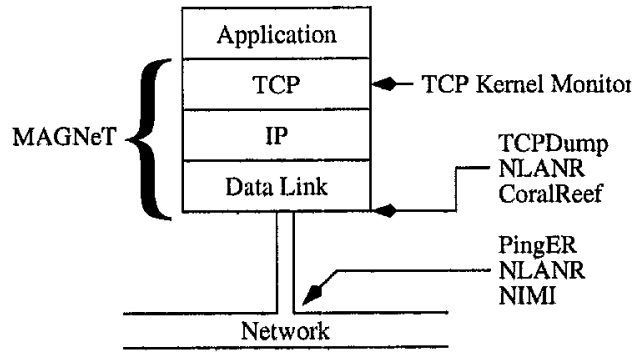

Fig. 1. Monitoring Points of Various Tools

So, researchers have not been testing the performance of network protocols using real, application-generated traffic traces; rather, they have used once-modulated (by the protocol stack) traffic traces as input, which are subsequently modulated a second time during the testing of the protocol. If the differences between application-generated traces and networkcaptured traces are negligible, such a simplification is acceptable. However, as we will show in this paper, the differences in the traces are substantial, indicating that the protocol stack adversely modulates the application-generated traffic patterns. This observation may invalidate the empirical results gathered from the performance evaluation of network protocols over the last decade because many researchers used network-captured traces rather than application-generated traces to drive their experiments.

What network researchers need are traces of real, application-generated traffic. Therefore, this paper describes a Monitor for Application-Generated Network Traffic (MAG$\mathrm{NeT}$ ) that captures traffic generated by the application rather than in the network.

\section{B. Related Work}

As shown in Fig. 1, MAGNeT differs from tcpdump-like measurement tools in that it can monitor traffic at the application level, (i.e., before it traverses the protocol stack), and throughout the entire protocol stack, as well as traffic entering and leaving the network. The only other measurement tool that makes similar measurements is the TCP kernel monitor from Pittsburgh Supercomputing Center [17].

MAGNe'T differs from the TCP kernel monitor in at least three ways. First, MAGNeT, although described in this paper as only a monitor for application-generated traffic (i.e., the interface between the application layer and TCP layer), can be used anywhere in the protocol stack. Second, MAGNeT monitors a superset of the data that the TCP kernel monitor does. Third, MAGNeT is implemented on the Linux operating system whereas PSC's TCP kernel monitor works on NetBSD. 


\section{Software ARChitecture}

Developing MAGNeT presents two design challenges. First is the issue of accurate time measurement. MAGNeT uses the CPU cycle counter available in modern microprocessors to record timestamps with cycle-level granularity. This functionality relies on code in the architecture-specific Linux code base but should be present on most Linux platforms.

The second, and more difficult, challenge requires that MAGNeT run on machines in a production environment in order to obtain realistic application-traffic traces. Hence, MAGNeT must incur minimal overhead so that the end user is not impacted and so that the application-generated traffic stream is not adversely perturbed. This latter consideration requires that any processing or filtering of the captured data be performed off-line; reducing or filtering the data in real-time not only adds an intolerable performance overhead but also adversely perturbs the application-generated traffic stream.

We address these design issues in our MAGNeT software distribution ${ }^{1}$, which consists of several user-application programs as well as modifications to the Linux kernel. The primary functionality of MAGNeT is contained in a patch to the Linux kernel. The patch creates a circular buffer in kernel memory and places calls throughout the networking stack to record appropriate information as data traverses the stack. A user-space program periodically empties this kernel buffer, saving the binary data to the disk. For post-processing the data, a set of data-analysis tools translates the binary data into machine- and human-readable form. Finally, a library of scripts to enable automated data collection from a set of hosts completes the MAGNeT package.

Fig. 2 illustrates the operation and dataflow of MAGNeT at a high level. Unmodified applications (that run on the test system) periodically make send () and recv () system calls to send and receive network traffic. These calls eventually make use of TCP, IP, or other network protocols in the kernel to transfer data on the network. For systems running MAGNeT, each time a send (), recv (), or network protocol call is made, an accompanying call is made to magnet-add (). This procedure saves relevant data to a circular buffer in kernel space, which is then saved to disk by the user-level application program magnet-read. The details of each of these steps is discussed below.

\section{A. MAGNeT in Kernel Space}

The MAGNeT kernel patch adds several functions to the Linux 2.4 kernel. The function magnet_add () adds a data point to a circular buffer that is guaranteed to always be in kernel memory. This function can be called virtually anywhere in the protocol stack; it is optimized so that each instrumentation call uses as few resources as possible. In addition, a new / proc item is added to the file space at $/$ proc/net/magnet. This file may be read by any user to determine the current state and parameters of the MAGNeT kernel code.

\footnotetext{
${ }^{1}$ The MAGNeT software distribution is currently undergoing alpha testing at LANL. A beta prototype will soon be publicly available from ht tp: / / ww . lanl.gov/radiant.
}

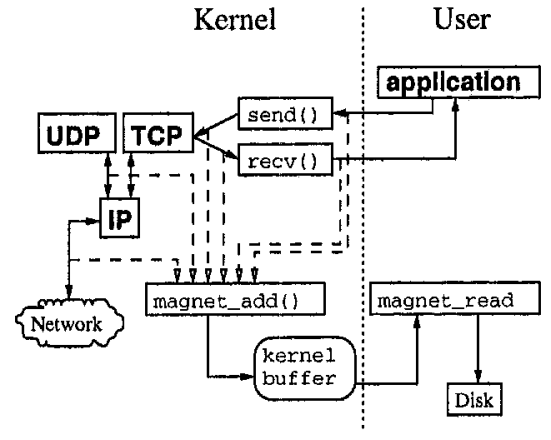

Fig, 2. Overview of MAGNeT Operation

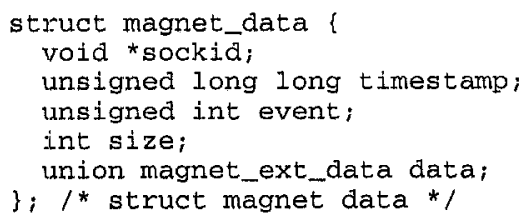

Fig. 3. The MAGNeT Instrumentation Record

\section{A.1 Instrumentation Record}

Fig. 3 shows the MAGNeT instrumentation record, the data structure that magnet_add () adds to the kernel buffer at each instrumentation point. sockid is a unique identifier for each connection stream, thus giving MAGNeT a way to separate data traces into individual streams while protecting the privacy of the application and user. The timestamp field is a CPU cycle counter that is used to synchronize MAGNeT events. Valid values for the event field (e.g., MAGNET_IP_SEND) indicate the type of event a particular record refers to. si ze contains the number of bytes transferred during a specific event. The data field (a optional field selected at kernel compile time) is a union of various structures in which information specific to particular protocols can be stored. This field provides a mechanism for MAGNeT to record protocol state information along with event transitions.

\section{A.2 Instrumented Events}

The MAGNeT kernel patch instruments the general sockethandling code, the TCP layer, and the IP layer. Other protocols can be easily instrumented by adding new MAGNeT event codes and placing calls to magnet_add () at appropriate places in the protocol stack.

There exists a possibility that the space in the fixed-sized, circular kernel buffer may be exhausted before the user-space program is able to read the records it contains. However, MAGNe'T will not overwrite any recorded data. This is accomplished by using the times tamp field of the instrumentation record as a synchronization flag between MAGNeT userand kernel- processes. Before writing to a slot in the circular buffer, the MAGNeT kernel code checks the value of the timestamp field for that slot. A value other than zero indicates that the slot has not yet been copied to user space and that the kernel buffer is full. In this case, the kernel code increments a count of the number of instrumentation records that 
could not be written due to the buffer being full. After the userlevel application reads a record from the buffer, it writes a zero to the timestamp field to signal to the kernel that the slot is available. Once buffer space becomes available, the kernel writes a special instrumentation record with an event type of MAGNET_LOST and with the size field set to the number of instrumentation records dropped.

Our experience to date indicates that while dropped instrumentation records are possible, they rarely occur during the monitoring of actual users. (See Section III-B.3 for details.)

\section{B. MAGNeT in User Space}

The user-level interface to MAGNeT consists of three application programs (magnet-read, magnet-parse, and mkmagnet), a special device file to facilitate kernel-user communication, and a collection of automating scripts. The application program magnet-read saves data from the kernel's buffer to a disk file and magnet-parse translates the saved data into an understandable format. mkmagnet is a small utility program to create the files that magnet-read requires to operate. The scripts included with the MAGNeT distribution allow the operation of MAGNeT to be fully automated and transparent to the end user.

\section{B.1 magnet-read, mkmagnet, magnet-parse}

To minimize the potential for record loss when MAGNeT reads the instrumentation records from the circular buffer in the kernel and writes them to disk, we map the circular buffer from kernel space into user space via a special "shared memory" device file. With this mapping in place, no additional kernel code is executed; the application program may simply read the shared memory and write it to disk. This transparency is accomplished by the program magnet-read, which copies the records by linking the shared memory region to a pre-existing file via the kernel's memory-mapped I/O system. The mkmagnet application creates and initializes the aforementioned file prior to being mapped into memory. The last tool, magnetparse, reads data collected by magnet-read and dumps a tab-delimited ASCII table of the collected data for further processing.

\section{B.2 Automating Scripts}

Two shell scripts in the MAGNeT distribution, magnet. cron and magnet. copy, provide examples of how to create an automated, transparent, application-monitoring environment on a campus network. magnet. cron is the overall MAGNeT management script, which should be executed upon initial boot, and at some interval (e.g., daily) during system operation. When run, it ensures that the proper device file exists and that a file has been created on disk with mkmagnet. It then starts magnet-read.

\section{MAGNeT Timestamps}

To ensure the greatest accuracy possible, MAGNeT uses the cycle counter available on contemporary microprocessors as the source of its timestamps. MAGNeT obtains this information via the kernel's getcyclecounter () function, which keeps the MAGNeT code hardware-independent. Given the
TABLE I

MAGNET VS, tcpdump

\begin{tabular}{|l|c|c|c|}
\hline Configuration & $\begin{array}{c}\text { Throughput } \\
(\mathrm{Kb} / \mathrm{s})\end{array}$ & $\begin{array}{c}\text { Send CPU } \\
(\%)\end{array}$ & $\begin{array}{c}\text { Receive CPU } \\
(\%)\end{array}$ \\
\hline Linux 2.4.3 & $94.1 \pm 0.0$ & $15.2 \pm 0.1$ & $33.5 \pm 0.1$ \\
MAGNeT & $94.1 \pm 0.1$ & $16.9 \pm 0.2$ & $33.5 \pm 0.1$ \\
magnet-read/rcv & $90.8 \pm 0.8$ & $20.7 \pm 0.3$ & $34.4 \pm 1.0$ \\
magnet-read/snd & $90.7 \pm 0.9$ & $23.7 \pm 1.7$ & $32.4 \pm 0.4$ \\
tcpdump/rcv & $89.4 \pm 1.5$ & $18.0 \pm 0.4$ & $59.8 \pm 0.9$ \\
tcpdump/snd & $89.4 \pm 0.8$ & $45.0 \pm 0.6$ & $31.9 \pm 0.3$ \\
\hline
\end{tabular}

(a) $100 \mathrm{Mbs}$ (Fast Ethernet)

\begin{tabular}{|l|c|c|c|}
\hline Configuration & $\begin{array}{c}\text { Throughput } \\
(\mathrm{Kb} / \mathrm{s})\end{array}$ & $\begin{array}{c}\text { Send CPU } \\
(\%)\end{array}$ & $\begin{array}{c}\text { Receive CPU } \\
(\%)\end{array}$ \\
\hline Linux 2.4.3 & $459.5 \pm 1.6$ & $61.0 \pm 0.3$ & $82.4 \pm 0.2$ \\
MAGNeT & $452.5 \pm 1.8$ & $63.0 \pm 0.4$ & $82.6 \pm 0.3$ \\
magnet-read/rcv & $444.3 \pm 1.7$ & $62.4 \pm 0.3$ & $82.0 \pm 0.3$ \\
magnet-read/snd & $440.2 \pm 2.1$ & $63.1 \pm 0.5$ & $81.1 \pm 0.4$ \\
tcpdump/rcv & $290.7 \pm 15.6$ & $36.1 \pm 2.0$ & $91.5 \pm 0.5$ \\
tcpdump/snd & $343.2 \pm 18.7$ & $93.2 \pm 0.5$ & $64.1 \pm 3.3$ \\
\hline
\end{tabular}

(b) $1000 \mathrm{Mbs}$ (Gigabit Ethernet)

speed of the processor, the difference between two cycle counts can be converted to elapsed time. Thus, the first record created by MAGNeT is of type MAGNET_SYSINFO and the size field of this record contains the processor clock speed in $\mathrm{kHz}$.

\section{MAGNeT Performance Analysis}

In this section, we demonstrate MAGNeT's ability to record application and network-stack events without adversely perturbing the traffic stream or application behavior. We also use MAGNeT-collected data to show significant differences between an application's network demands and the actual traffic delivered to the network.

\section{A. Experimental Method}

As an indication of how much MAGNeT perturbs application-generated network traffic, we measure the maximum data rate between a sender and receiver as well as the CPU utilization. In addition, we measure the overhead of running tcpdump as a point of comparison. In total, we run six different configurations on 100-Mbps and Gigabit Ethernet networks, as shown in Table I.

We conduct our tests between two identical dual $400 \mathrm{MHz}$ Pentium IIs with $100 \mathrm{Mbps}$ or $1000 \mathrm{Mbps}$ Ethernet card (connected via an Extreme Networks Summit 7 i switch) and configure MAGNeT to record application send () and recv () socket calls as well as TCP and IP events. MAGNeT uses the default 256KB kernel buffer to store event records.

To generate the workload, we run netperf [18] on the sender, ${ }^{2}$ transmitting data as fast as possible. We minimize the amount of interference in our measurements by eliminating all other network traffic and minimizing the number of processes running on the test machines to netperf and a few essential Linux services.

\footnotetext{
${ }^{2}$ The command used was "netperf $-\mathrm{P} \quad 0$ - $\mathrm{C}$ [1ocal CPU index -C $\{$ remote CPU index\} -H \{hostname\}"
} 


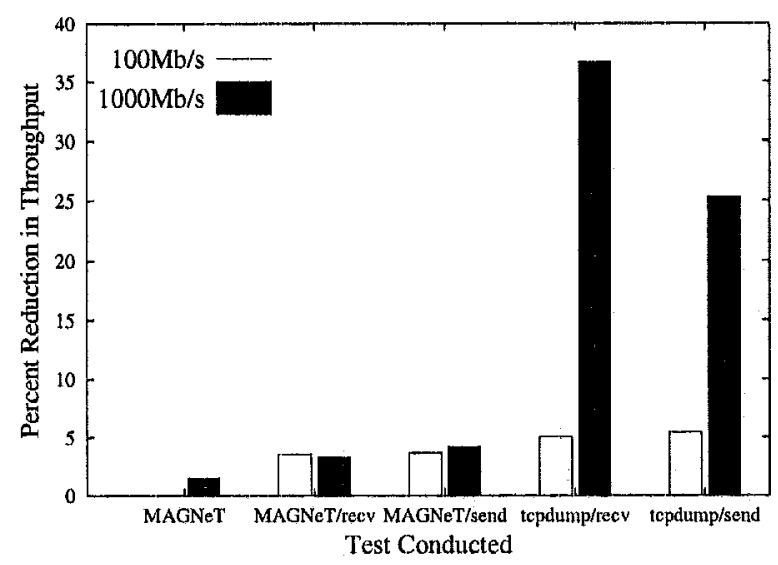

Fig. 4. Percent Reduction in Network Throughput

\section{B. Performance}

While no monitoring system can be completely transparent to the workload being monitored, MAGNeT is designed to have minimal impact on overall network throughput. Table I shows how MAGNeT performs in comparison to tcpdump. Along with the mean, the width of the $95 \%$ confidence interval is given. Figures 4 and 5 present this data graphically.

By default (and as used in our experiments), tcpdump stores the first 68 bytes of every packet. Our configuration of MAGNeT, on the other hand, stores 96 bytes for each packet. ${ }^{3}$

\section{B.1 Network Throughput}

The kernel-resident portion of MAGNeT is always executing, regardless of whether information is being saved to disk or not. The first pair of bars in Fig. 4, labeled "MAGNeT," shows only a small penalty when no data is being saved to disk. The next two pairs of bars show that MAGNeT incurs less than a $5 \%$ reduction in network throughput when magnet-read runs on either the receiver or sender. Furthermore, the penalty is nearly constant regardless of network speed. In contrast, while tcpdump incurs roughly the same penalty as MAGNeT over $100 \mathrm{Mbps}$ networks, the penalty increases to $25 \%-35 \%$ of total throughput at $1000 \mathrm{Mbps}$. Thus, MAGNeT scales better with increasing link speeds than tcpdump.

\section{B.2 CPU Utilization}

Next, we compare MAGNeT's and tcpdump's CPU utilization as reported by netperf. ${ }^{4}$ Each bar in Fig. 5 reflects the percentage increase in CPU utilization averaged over both the sender and the receiver. In this case, MAGNeT also performs better than tcpdump, which is not surprising since tcpdump makes system calls from user space (thus incurring a context switch) for every packet while MAGNeT executes primarily within the kernel. What may be surprising is that both MAGNeT's and tcpdump's overhead appears to decrease when run

\footnotetext{
${ }^{3}$ Although MAGNeT's record size is only 24 bytes per event, our configuration of MAGNeT instruments the events at each protocol layer in the stack, resulting in four events per packet (or $24 \times 4=96$ bytes per packet).

${ }^{4}$ This version of netper $f$ measures CPU utilization via an "idle" loop in a very low-priority process (which theoretically should only run when the system is otherwise idle)
}

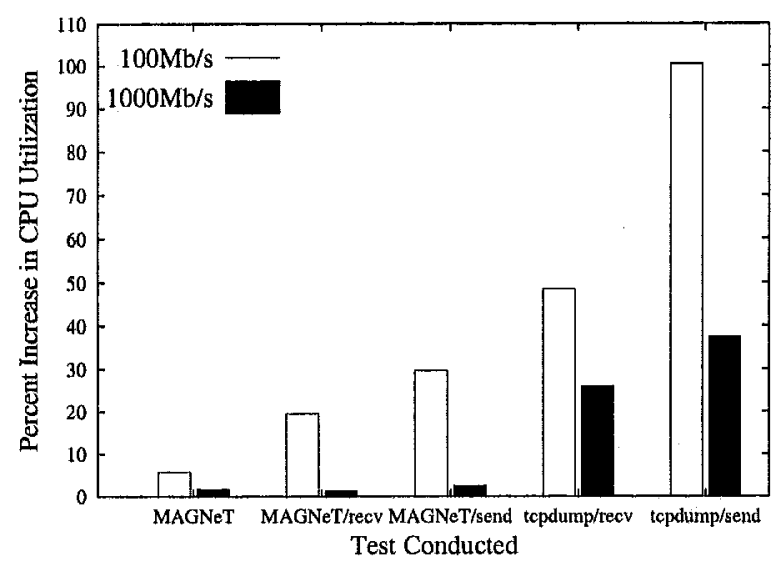

Fig. 5. Average Percent Increase in CPU Utilization

on the faster network. This is due to interrupt coalescing, i.e., the network interface cards accumulate several incoming packets before interrupting the CPU. Thus, the average overhead of servicing interrupts is greatly reduced. Had interrupt coalescing been disabled, the average CPU utilization for both MAGNeT and tcpdump would have increased.

\section{B.3 Event Loss}

During our testing, analysis of the MAGNeT-collected data revealed that MAGNeT occasionally loses events at high network utilization. For the $100 \mathrm{Mbps}$ trials, MAGNeT lost less than than $3.2 \%$ of the total events; for the $1000 \mathrm{Mbps}$ tests, the loss rate approached $15 \%$. These losses are due to the $256 \mathrm{~KB}$ circular buffer in the kernel filling before magnet-read is able to drain it.

Two methods exist for reducing the loss rate: (1) increasing the kernel buffer size and/or (2) reducing the time magnetread waits before draining the kernel buffer. Fig. 6 shows how changing the kernel buffer size or magnet-read delay affects event-loss rate on a $100 \mathrm{Mbps}$ network. (Note: All other tests discussed in this paper used MAGNeT's default values for these two parameters - 256KB kernel buffer with magnetread's automatically calculated delay time.)

Increasing the size of the kernel buffer dramatically reduces MAGNeT's potential for event loss, down to virtually no lost events under any network load with a $1 \mathrm{MB}$ buffer. However, because this buffer is pinned in memory, a large buffer also reduces the amount of physical memory available to the kernel and applications. We chose $256 \mathrm{~KB}$ to be the default buffer size to reduce both CPU utilization and physical memory consumed (i.e., to reduce the invasiveness of the monitor).

Another method for reducing event loss entails adjusting the amount of time magnet-read sleeps before draining the kernel buffer. However, shorter sleep times create more work (in terms of CPU usage, and possibly, disk write activity), and thus may interfere with the system's normal use in a production environment.

The default sleep-time is computed as the average amount of time it takes to fill the kernel buffer on a $100 \mathrm{Mbps}$ network. This heuristic was chosen because it provides relatively low event-loss rates without significantly impacting the user. 


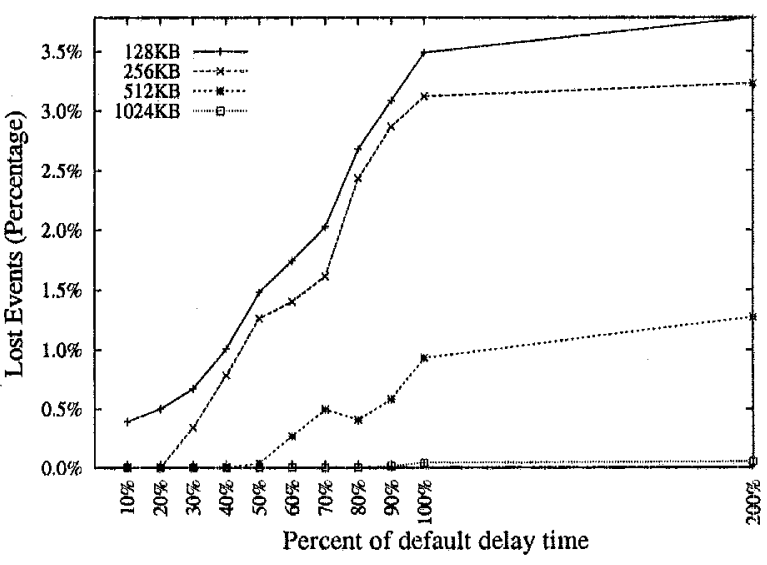

Fig. 6. MAGNeT's Event-Loss Rate, 100Mbs Ethernet

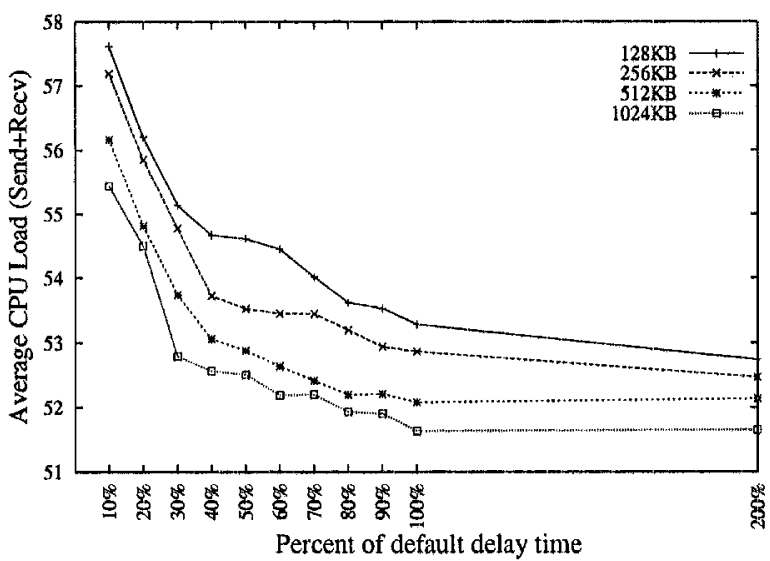

Fig. 7. MAGNeT's Average CPU Utilization, 100Mbs Ethernet

Command-line options are provided in magnet-read to adjust the delay. Fig. 7 shows the increase in average CPU utilization for different sleep times and buffer sizes with MAG$\mathrm{NeT}$ running on the sending machine. While these differences appear minor, even a small decrease in available CPU cycles can have a dramatic effect on application run-time and communication patterns (and thus, increased CPU utilization may result in large perturbations of the monitored traffic).

By comparison, tcpdump also loses information, approximately $15 \%$ on a $100 \mathrm{Mbps}$ network (for the extreme load conditions of our tests). This loss rate increases rapidly with higher network speeds because tcpoump does no buffering.

In summary, if MAGNeT's loss rate is too high, it can be adjusted to an acceptable level via the mechanisms discussed above; tcpdump lacks such adjustability. For example, to drop MAGNeT's loss rate to $0.5 \%$ while using the default $256 \mathrm{~K}$ buffer, we adjust the magnet-read delay time to $35 \%$ of its original value (Fig. 6). According to Fig. 7, this would increase the overall CPU utilization from $53 \%$ to $55 \%$. This is still less than the $60 \%$ CPU utilization seen with tcpdump.

\section{Network Perturbation}

From the measurements presented in this section, MAGNeT clearly performs as efficiently as tcpdump on conterm- porary networks and more readily scales to higher-speed networks. In addition, by adding CPU cycle-counter code around magnet-add () and relevant areas of magnet-read, we are able to compute the number of cycles, on average, that MAGNeT consumes while recording a single event. This value is of interest in comparison to the minimum interarrival time for packets on the physical network. For instance, on a $100 \mathrm{Mbps}$ Ethernet, a 40-byte Ethernet packet - the size of an "empty" TCP packet - will arrive no faster than (40 bytes * 8 bits/byte) $/ 100 \mathrm{Megabits} / \mathrm{second}=3.2 \mu \mathrm{sec}$. Our conservative tests indicate that magnet-add () requires 556 cycles, on average, per recorded event while magnet-read requires 425 cycles. Thus, on our $400-\mathrm{MHz}$ machines, MAGNeT takes $(556+425)$ cycles $/(400000000$ cycles $/$ second $)=2.4 \mu \mathrm{sec}$ to record a single event. Since this is less time than it takes a minimal TCP packet to arrive or to be sent, the MAGNeT-induced disturbances into the traffic stream should be quite small.

\section{Design of tcpdump vs. MAGNeT}

The performance numbers in this paper reflect a difference in design philosophies between tcpdump and MAGNeT. tcpdump is a purely user-level, network-inspection tool, which can be (and has been) relatively easily ported to a large number of different operating systems. Thus, many of the design tradeoffs in tcpdump favor portability over pure performance. In particular, libpcap, the packet capture library created for tcpdump and used by other network monitors, such as CoralReef, reflects this approach. The exact method used to intercept network packets varies depending on the features available in the root operating system, but it always involves a system call or other facility to cause a switch into kernel mode and a copy of memory from the kernel to the user-level program. This call-and-copy is repeated for every packet traveling across the interface being monitored. At high network speeds, the overhead of copying each individual packet between kernel and user space becomes a significant burden. MAGNeT benefits from having code embedded in the kernel to aggregate multiple network packets into a single space which then is copied in bulk, thus amortizing the cost of the copy over multiple packets. This approach incurs less overhead but is not as portable as libpcap's method.

\section{MAGNET IN THE REAL WORLD}

We initially developed MAGNeT to investigate differences between traffic generated by an application and that same traffic after modulation by the protocol stack, i.e., when the traffic hits the network. Thus far, we have observed many situations where significant differences exist. For instance, Fig. 8 shows a one-second trace of an FTP session that was taken one minute into sending a Linux 2.2.18 kernel bzipped tar file from our facilities in Los Alamos, NM to a location in Dallas, TX - a trip of 30 hops across the Sprintlink backbone fabric. This graph shows a dramatic difference (in both packet size and inter-packet spacing) between application-generated traffic patterns and the traffic actually delivered to the network.

Network researchers routinely test new network and protocol designs using traces of traffic seen in current networks rather than the real traffic demands of applications. That 


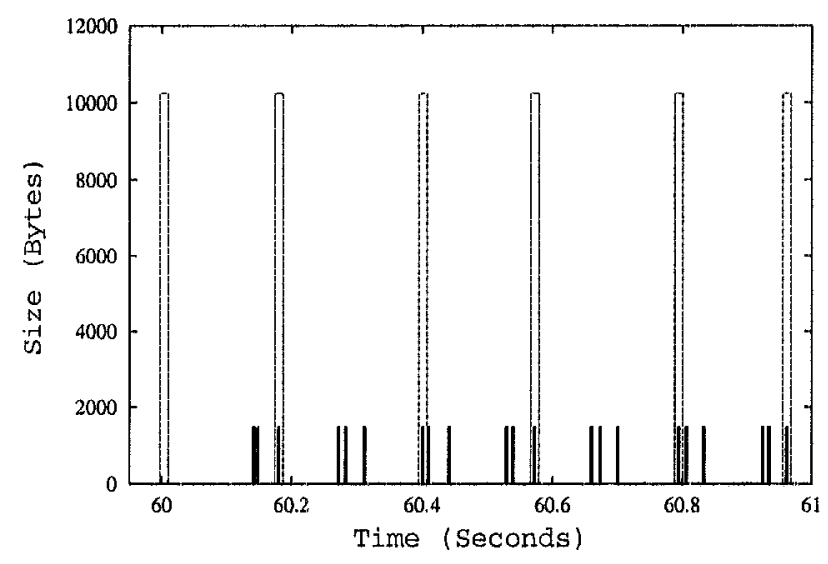

Delivered to network - Application sendo call -..........

Fig. 8. MAGNe'T FTP Trace

TABLE II

EFFECT OP MULTIPLE TCP.STACK TRAVERSALS

\begin{tabular}{|l|c|c|}
\hline Trial & Data Size & $\begin{array}{c}\text { Interpacket Spacing } \\
(\mathrm{sec})\end{array}$ \\
\hline Application & 3284 & 0.124 \\
1st TCP stack & 1016 & 0.045 \\
2nd TCP stack & 919 & 0.037 \\
3rd TCP stack & 761 & 0.079 \\
4th TCP stack & 723 & 0.122 \\
\hline
\end{tabular}

is, new designs are tested with network-delivered traffic, not application-generated traffic. Thus, differences such as those shown in Fig. 8 may have significant impact on the accuracy of such tests. For example, if the network-delivered traffic pattern from the above example is taken as the input to test the performance of an enhanced TCP stack (as would be the case if a tcpdump trace of the FTP session was used as input to a network simulation), the packet sizes and inter-packet spacing are once again modulated, as illustrated in Table II (in the row labeled "lst TCP stack"). The remainder of the table shows the effect of repeatedly using TCP output streams as inputs to subsequent TCP stacks. ${ }^{5}$

\section{CONCLUSION}

Current traffic libraries, network traces, and network models are based on measurements made by tcpdump (or similar tools such as PingER, NLANR Network Analysis Infrastructure, NIMI, and CoralReef). These tools do not capture an application's true traffic demands; instead they capture an application's demands after having been modulated by the protocol stack. Therefore, existing traffic libraries, network traces, and network models cannot provide any protocol-independent

\footnotetext{
${ }^{5}$ Such repeated use is possible when communicating between end hosts in different domains. For example, when sending data via FTP between hosts at Los Alamos National Laboratory (LANL) and Sandia National Laboratory (SNL), the FTP connection is actually broken up into three consecutive connections, i.e., (1) LANL end host to LANL firewall, (2) LANL firewall to SNL firewall, and (3) SNL firewall to SNL end host. Thus, the initial applicationgenerated traffic pattern that originated at the LANL end host goes through the protocol stack a total of six times (three sends and three receives).
}

insight into the actual traffic patterns of an application.

MAGNeT fills the above void by providing a flexible and low-overhead infrastructure to monitor network traffic virtually anywhere in the protocol stack. Using MAGNeT, we have shown that the traffic demands of applications are not accurately reflected by the traffic on the network wire because the protocol stack (i.e., TCP) adversely modulates the applicationgenerated traffic before it gets to the network wire. Hence, while current network models may accurately reflect current network-wire traffic, they are not useful in providing insight into application-generated traffic patterns nor in optimizing $a p$ plication communications.

The potential applications of MAGNeT include, but are not necessarily limited to, (1) constructing a library of traces of application-generated traffic, (2) verifying the correct operation of protocol enhancements to TCP, IP (also IPv6 and IPsec), or other protocols, (3) troubleshooting and tuning protocol implementations, and (4) security scanning. In this paper, we focused on the first application for MAGNeT - by providing a library of application-generated network traces, the network research community can drive their experiments (whether simulative and live) with real, application-generated traffic.

\section{REFERENCES}

[1] "tcpdump," http : / /www . tcpdump. org.

[2] W. Matthews and L. Cottrell, "The PingER Project: Active Internet Performance Monitoring for the HENP Community," IEEE Communications, May 2000.

[3] S. Kalidindi and M. Zekauskas, "Surveyor: An Infrastructure for Internet Performance Measurements," in INET'99 Proceedings.

[4] A.J. McGregor, H-W Braun, and J.A. Brown, "The NLANR Network Analysis Infrastructure," IEEE Communications, May 2000.

[5] V. Paxson, J. Mahdavi, A. Adams, and M. Mathis, "An architecture for large-scale internet measurement," IEEE Communications, 1998.

[6] CAIDA, "CoralReef Software Suite," ht $t p: / / w w w . c a i d a . o r g /$ tools/measurement/coralreef.

[7] W. Leland, M. Taqqu, W. Willinger, and D. Wilson, "On the Self-Similar Nature of Ethernet Traffic (Extended Version)," IEEE/ACM Transactions on Networking, vol. 2, no. 1, pp. 1-15, February 1994.

[8] K. Park, G. Kim, and M. Crovella, "On the Relationship Between File Sizes, Transport Protocols, and Self-Similar Network Traffic," in Proc. of the Int'l Conf. on Network Protocols, October 1996.

[9] V. Paxson and S. Floyd, "Wide-Area Traffic: The Failure of Poisson Modeling," IEEE/ACM Transactions on Networking, vol. 3, no. 3, pp. 226-244, June 1995.

[10] V. J. Ribeiro, R. H. Riedi, M. S. Crouse, and R. G. Baraniuk, "Multiscale Queuing Analysis of Long-Range Dependent Network Traffic," in Proc. of IEEE INFOCOM'O0, March 2000

[11] P. Tinnakornstisuphap, W. Feng, and I. Philp, "On the Burstiness of the TCP Congestion-Control Mechanism in a Distributed Computing System," in Proc. of the Int'l Conf. on Dist. Comp. Sys., April 2000.

[12] W. Feng and P. Tinnakornsrisuphap, "The Adverse Impact of the TCP Congestion-Control Mechanism in Heterogeneous Computing Systems," in Proc, of the Int'l Conf. on Parallel Processing, August 2000.

[13] W. Feng and P. Tinnakornsrisuphap, "The Failure of TCP in HighPerformance Computational Grids," in Proc. of SC 2000: HighPerformance Networking and Computing Conf., November 2000.

[14] P. Danzig and S. Jamin, "tcplib: A Library of TCP Internetwork Traffic Characteristics," http://irl.eecs.umich.edu/jamin/ papers/tcplib/tcplibtr.ps.z,1991.

[15] "The Internet Traffic Archive," http://1ta.ee.1bl.gov/html/ traces.html.

[16] A. Kato, J. Murai, and S. Katsuno, "An Internet Traffic Data Repository: The Architecture and the Design Policy," in INET'99 Proceedings.

[17] J. Semke, "PSC TCP Kernel Monitor," Tech. Rep. CMU-PSC-TR-20000001, PSC/CMU, May 2000.

[18] "Netperf," http: //www .netperf .org. 